\title{
Tracking Testing of Dynamic Errors for Linear Guideway Based on Position Sensitive Detector
}

\author{
Dazhong Guo ${ }^{1, a}$, Yuanyuan Zou ${ }^{2, b}$, Sijun Zhu ${ }^{3, \mathrm{c}}$, Mingyang Zhao ${ }^{4, \mathrm{~d}}$ \\ 1,2,3,4 Shenyang Institute of Automation, Chinese Academy of Sciences Shenyang 110016, China \\ adzguo@sia.cn, byyzou@sia.cn, ’sjzhu@sia.cn, ${ }^{\mathrm{d}} \mathrm{myzhao@sia.cn}$
}

Keywords: position sensitive detector, motion stability measurement, laser tracing measurement system

\begin{abstract}
The dynamic precision of linear guideway is very important performance for mechatronic equipments. The dynamic errors for the linear guideway of machine tools can reduce machining precision. In according with the advantage of high-speed response, high detection accuracy and non-contact measurement, position sensitive detector (PSD) is widely used in industrial dynamic measurement. In this paper an approach for measuring the motion stability based on PSD is proposed. The measurement system based on PSD is designed and the measurement errors are analyzed. The measurement results show the approach can measure accurately the motion stability of guideway in two dimensions with features of high precision.
\end{abstract}

\section{Introduction}

The dynamic precision of linear guideway is very important performance for mechatronic equipments, machine tools in particular. The dynamic errors for the linear guideway of machine tools can reduce machining precision ${ }^{[1]}$. Therefore tracking testing of dynamic errors for linear guideway plays an important role in analyzing and improving performance of machine tools, and the studying can provide theoretical basis of keeping precision.

The traditional measuring method can only measure one direction of position offset, and has poor precision usually. Position Sensitive Detector (PSD) is widely used in vibration measurements and precision displacement measurements due to its high-speed response, high position resolution and noncontact ${ }^{[2,3]}$, and it plays more and more important role in dynamic measurements and vibration measurements. An approach for measuring the dynamic errors of linear guideway in two dimensions based on PSD is proposed in this paper.

\section{Measuring principle and system structure}

The dynamic errors of the linear guideway has two directions of level and vertical, and the approach based on PSD in this paper can measure the two directions simultaneity of dynamic errors. The principle of measurement system is shown in Figure 1.

The whole measurement system is composed of a linear guideway, beam splitter, target mirror, laser interferometer. Tracking device and PSD. The target mirror is fixed in a movable part of linear guideway that will be measured, and the laser interferometer emits a light to the target mirror, the reflected light of the target mirror that has the information of the dynamic position goes through the beam splitter, and the light is divided into two beams of light by the beam splitter, one goes into the laser interferometer, another goes into the PSD. The X and Y direction position of light spot of the beam of measuring light on PSD can be measured by PSD, so that we can read the accurate value of the two direction position of the target mirror on the linear guideway, and PSD sends out the position information of the target mirror to tracking device on which PSD is fixed in real time. The purpose of tracking device is to maintain the light spot on the center of PSD. The demarcation of $\mathrm{X}$ and $\mathrm{Y}$ direction position on PSD is very important for the measuring precision before measuring. The approach to demarcate PSD is that the target mirror is fixed on standard linear guideway, and measuring the position by PSD and laser interferoment, the ratio of PSD and real position is calculated by a certain arithmetic, and the error compensation that the errors that are brought by air, temperature and background light is considered in addition. 


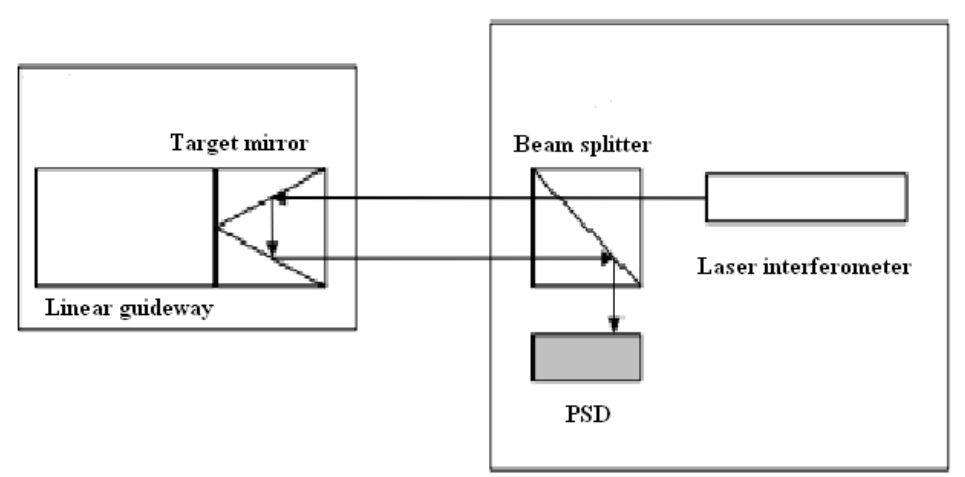

Fig. 1. Diagram of measurement principle

The two dimensions of PSD can measure two direction position errors of the light spot. The position of light spot on PSD can be calculated by the following expressions:

$$
\begin{aligned}
& \frac{\left(V_{X 2}+V_{Y 1}\right)-\left(V_{X 1}+V_{Y 2}\right)}{V_{X 1}+V_{X 2}+V_{Y 1}+V_{Y 2}}=\frac{2 x}{L_{x}} \\
& \frac{\left(V_{X 2}+V_{Y 1}\right)-\left(V_{X 1}+V_{Y 2}\right)}{V_{X 1}+V_{X 2}+V_{Y 1}+V_{Y 2}}=\frac{2 y}{L_{y}}
\end{aligned}
$$

where $V_{X 1}, V_{X 2}, V_{Y 1}, V_{Y 2}$ are converting voltage of the output current of electrode $X 1, X 2, Y 1$, $Y 2, L_{x}, L_{y}$ are the length of the direction $\mathrm{X}, \mathrm{Y}$ of PSD photoreceptor, and $x, y$ are the coordinate value of the light spot on PSD.

\section{Experiment and result analysis}

The measuring experiment is done in several velocities to measure the dynamic errors, and the result of the experiment is analysed.

The four repeat experiments are done at the speed of $10 \mathrm{~mm}$ per a second. The experimental results are shown as the following Figure 2, and the figure shows the direction $\mathrm{X}$ and $\mathrm{Y}$ position offset of PSD. Experimental results show that the measuring system has well position repeatability.

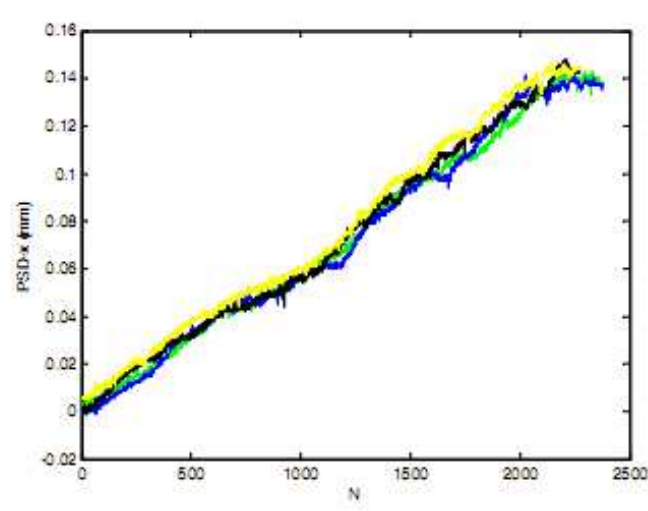

(a) PSD - $x$

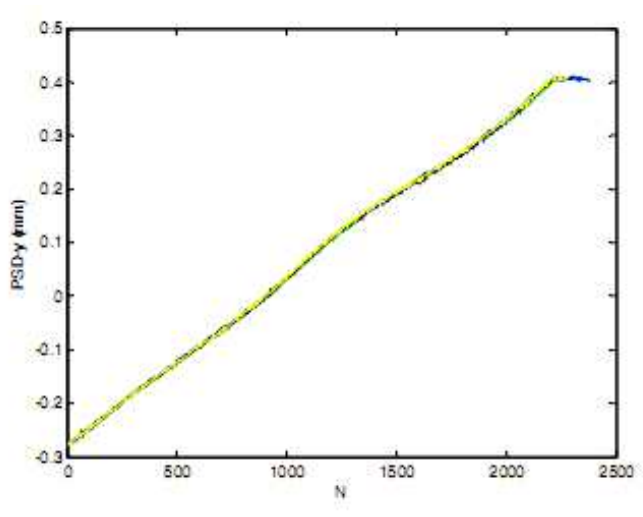

(b) PSD - y

Fig. 2. Results of repeatability experiment

The experiment of measuring motion stability is done at different speed. At first the straightness deviation of linear guideway is measured, and the measuring experiment is done in the quasi-static state, the experimental results of the direction X and Y position offset of PSD show as the following Figure 3. 

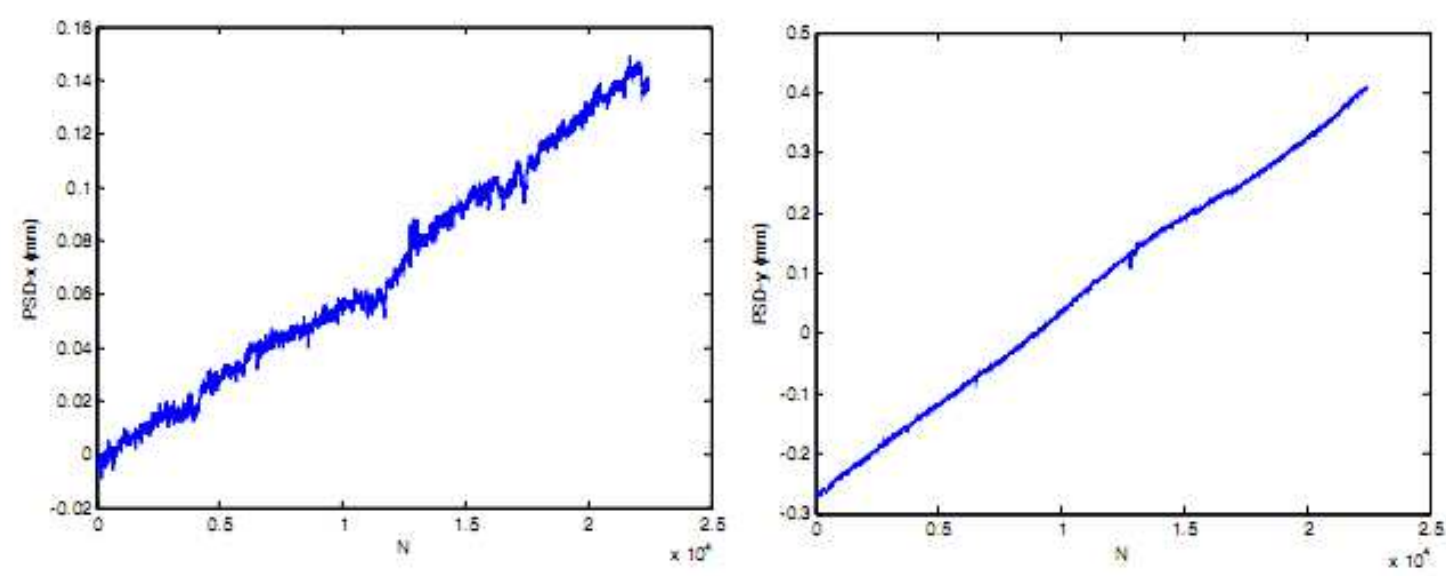

Fig. 3. Position displacement of quasi-static

The experiment of measuring the direction $\mathrm{X}$ and Y position offset of PSD is done at the speed of $30 \mathrm{~mm}$ per second, $50 \mathrm{~mm}$ per second, $100 \mathrm{~mm}$ per second. The experimental results of the direction $\mathrm{X}$ and $\mathrm{Y}$ position offset of linear guideway show as the following Figure 4.
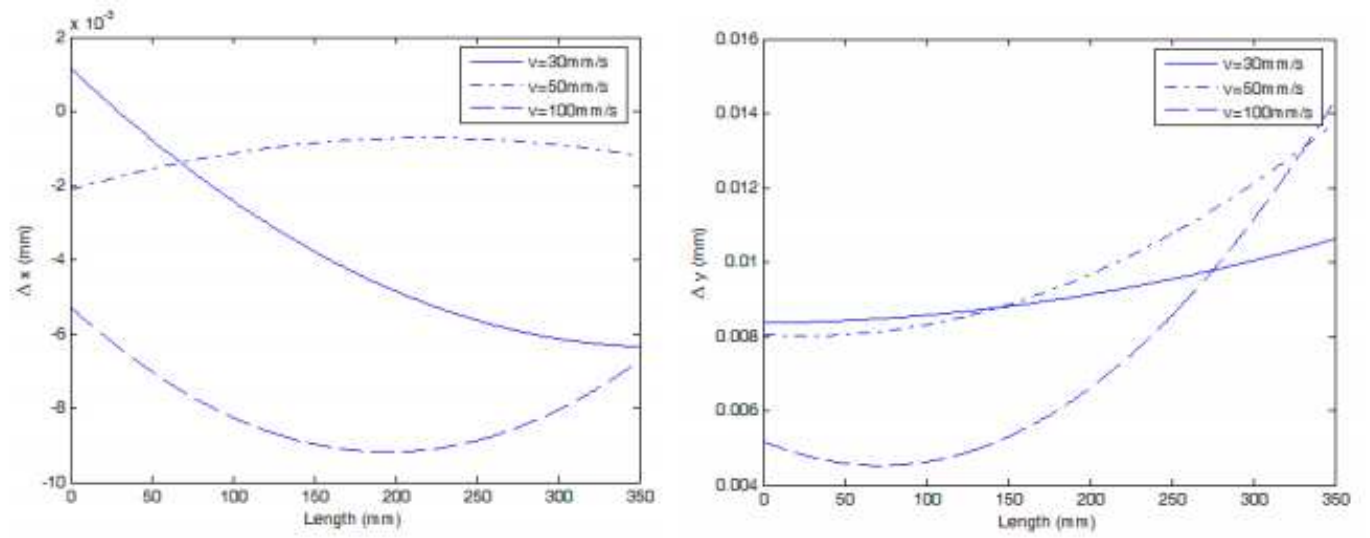

Fig. 4. Results of experiments at different speed

The errors of the experiment system mostly include the following aspects:

Straightness error of linear guide: Straightness error of linear guide can result in the measuring error of PSD. The real measuring results of PSD include straightness error of linear guideway and motion stability of linear guideway.

Positioning error of PSD: As the manufacturing error, it has different resistivity in the photosensitive surface of PSD, and the output of PSD has nonlinear error. Therefore the output results of PSD should be corrected in time.

Infection of background light: As broad spectral response range of PSD, the measuring results of PSD can be effected by the background. We can make use of light filter to restraining the infection of background light.

Infection of air refractive index: The light can be effected in air by air refractive index. We should calibrate the air refractive index before measuring experiment, and keep the air of experiment clean and prevent the pollution of air into the measurement range.

Environmental vibration: Environmental vibration can cause the measurement error. For restraining environmental vibration it can be considered to use of vibration isolation device in the measurement system.

\section{Conclusion}

In this paper the measurement system based on PSD is designed to measure the motion stability of two dimensions guideway. The measurement principle and approach is introduced, and measurement errors are analyzed in detail. The position error is caused by many reasons, for example straightness 
error of linear guide, positioning error of PSD, infection of background light, infection of air refractive index, and environmental vibration. If these reasons are effectively compensated, the measurement system based on PSD can measure the guideway stability in high precision.

\section{References}

Reference an article:

[1] Huang Xin. Research on Engineering and Analysis on Performance of Linear Rolling guide. Beijing: Higher Education Press, 2004. 3-21.

[2] Zhang Daxing, Jia Wei, Guan Bolin, Investigation for vibration measurement and control based on photoelectric displacement detectional, Journal of Electronic Measurement and Instrument, 2009, 23, 163-166.

[3] Su Haiyan, Luo Zushun, Yang Yongcai, The Study of Photoelectric Online Measurement for Axial Vibration Based on LabVIEW, Chinese Journal of Scientfic Instrument, 2004, 25, 26-28.

[4] Guo Lifeng, Zhang Guoxiong, Gong Qiang, Zheng Qi, Application of 2-D Position Sensitive Detector in Spatial Straightness Measurement of Guide Rail, Transactions of Tranjin University, 2005, 11(1), 44-48.

[5] Chen Dongyan, Lin Bin, Chen Yuqing, Development and Progressing of Position Sensitive Detector, Optical Instruments, 2004, 26(4), 68-71.

[6] Zhu Mingzhu, Chen Peifeng, Zhou Baoyu, Zeng Wei, The Error Analysis and Experimental Study on PSD Used in Laser Position Detection, Optoelectronic Technology \& Information, 2006, 19(3), 10-14. 